\title{
Comparación de dos cuestionarios autoaplicables para la detección de síntomas depresivos en gestantes
}

\author{
Comparison of two self report questionnaires for depressive symptoms detection \\ in pregnant women
}

\author{
Nelly Lam ${ }^{1,2}$, Hans Contreras ${ }^{3}$, Elizabeth Mori³, Fátima Cuesta ${ }^{3}$, César Gutiérrez $^{4}$, \\ Miluska Neyra ${ }^{3}$, Enrique $\mathrm{Gil}^{3}$, Giulianna Córdova ${ }^{3}$ \\ ${ }^{1}$ Investigador del Instituto de Investigaciones Clínicas. Facultad de Medicina, Universidad Nacional Mayor de San Marcos. Lima, Perú. \\ ${ }^{2}$ Miembro del Programa Permanente de Capacitación para la Atención Integral de las Víctimas de Violencia. Facultad de Medicina, Universidad Nacional \\ Mayor de San Marcos. Lima, Perú. \\ ${ }^{3}$ Sociedad Estudiantil para el Fomento de la Investigación y las Neurociencias (SEFIN). Facultad de Medicina, Universidad Nacional Mayor \\ de San Marcos. Lima, Perú. \\ ${ }^{4}$ Departamento Académico de Medicina Preventiva y Salud Pública. Facultad de Medicina, Universidad Nacional Mayor de San Marcos. Lima, Perú.
}

\section{Resumen}

Objetivo: Evaluar el comportamiento de dos cuestionarios autoaplicables para detectar síntomas depresivos en una misma población de gestantes. Diseño: Estudio observacional transversal analítico. Institución: Instituto Nacional Materno Perinatal de Lima, Perú. Participantes: Trescientos siete gestantes sin antecedente psiquiátrico diagnosticado, atendidas en la Unidad de Medicina Fetal en mayo y junio de 2008. Instrumentos: Escala de depresión posnatal de Edimburgo (EPDS) y el inventario de la depresión de Beck (BDI-II). Principales medidas de resultados: Medias, consistencias internas y coeficiente de Pearson. Resultados: Las medias para ambas escalas fueron altas: 11,36 para EPDS (rango: 0 a 26) y 15,96 para BDI-II (rango: 0 a 47). Las consistencias internas fueron altas (alfa de Cronbach 0,826 para EPDS y 0,815 para BDI-II). El coeficiente de correlación de Pearson entre las escalas fue de $0,541(p<0,001)$. Conclusiones: Tanto los resultados de la escala de depresión posnatal de Edimburgo como los del inventario de la depresión de Beck presentaron consistencia interna alta y correlación aceptable. Los elevados puntajes promedios de ambos cuestionarios reflejan una prevalencia alta del trastorno depresivo en las gestantes estudiadas, por lo cual sugerimos incluir dentro de los exámenes de rutina de la Unidad de Medicina Fetal, del Instituto Nacional Materno Perinatal, el despistaje de trastornos mentales, en especial la depresión gestacional.

Palabras clave: Síntomas depresivos; embarazo; depresión posnatal.

\begin{abstract}
Objective: To determine the ability of two self-report questionnaires to detect depressive symptoms in the same pregnant women population.

Design: Analytic, observational, cross sectional study.

Setting: Instituto Nacional Materno Perinatal de Lima, Peru. Participants: Three hundred and seven pregnant women with no psychiatric history diagnosis attended during May-June 2008.

Instruments: Edinburgh postnatal depression scale (EPDS) and Beck depression inventory (BDI-II).

Results: Both scales had high average: 11,36 for EPDS (range: 0-26) and 15,96 for BDI-II (range 0-47). Internal consistencies were high (Cronbach's alpha for EPDS was 0,826 and 0,815 for BDI-II). The Pearson correlation coefficient between both questionnaires was $0,541(p<0,001)$.

Conclusions: The study shows that both Edinburgh postnatal depression scale and beck depression inventory present high internal consistency and acceptable correlation. High averages found would show high probability in finding a depressive disorder in the mothers studied. Consequently we suggest a routine mental disorder screening at the Unidad de Medicina Fetal (Department of Fetal Medicine).
\end{abstract}

Key words: Symptoms, depression; pregnancy; depression, postpartum.

\section{INTRODUCCIÓN}

La investigación acumulada en el devenir de la última década sugiere que los problemas de salud mental y, en especial la depresión durante la gestación, son factores de riesgo independientes que aumentan la morbilidad y la mortalidad tanto materna como fetal ${ }^{(1)}$, asociándose en este último caso con problemas neurológicos y psiquiátricos en la infancia y la adolescencia ${ }^{(2-5)}$.

Por ello, la Sociedad Americana de Ginecología y Obstetricia sugirió que en los centros encargados de velar por la salud materno-perinatal, durante la consulta prenatal, se debería prestar especial atención al tamizaje de las mujeres gestantes con este tipo de trastorno. Para ello se propuso la utilización de los cuestionarios autoaplicables (CA) que, en el curso de cierto número de preguntas, evalúan la presencia de síntomas depresivos. En el Perú, la utilización de los CA no es usual en la práctica médica, pero sí en la investigación ${ }^{(6-8)}$, ya que desde hace algunos años ha sido sumado a los aportes científicos extranjeros, demostrando la importancia de considerar a la depresión gestacional como lo que es, no un traspié del 'ser psicológico' sino un desorden multicausal, de raigambre eminentemente biológica (epigenética) y social (cinética) y por tanto psíquica consciente, con consecuencias para la madre como para el ser humano en formación ${ }^{(9-11)}$. Recordemos que, como plantea la Teoría Psicobiológica Dialéctica, la vida empieza en la concepción y no en el nacimiento, al mismo tiempo que es síntesis de múltiples determinaciones ${ }^{(12)}$.

Sin embargo, y volviendo a nuestro campo de estudio, para que un CA pueda ser utilizado en población gestante debe demostrar cierto grado de validez como instrumento. Los únicos instrumentos validados en el campo de la salud mental perinatal son la Escala de Depresión Postnatal de Edimburgo (EPDS) y el Inventario de la Depresión de Beck (tanto en su primera versión ${ }^{(13)}$, de 1961, que en nuestro estudio no tomaremos en cuenta, como en su segunda versión ${ }^{(14)}$, 
de 1996, que la consideraremos para el presente estudio) (Tabla 1). En el Perú, solo la EPDS ha sido validada ${ }^{(6)}$, aunque no en población gestante sino durante el posparto.

En la población gestante, la EPDS ha sido validada en Estados Unidos ${ }^{(15)}$, en Francia ${ }^{(16)}$, en Nigeria ${ }^{(17)}$ y en el Reino Unido ${ }^{(18)}$. Recientemente, en un estudio realizado en Taiwán ${ }^{(19)}$, se validó conjuntamente las dos escalas, de lo cual se pudo confirmar la presunción planteada por Bennett y col. ${ }^{(20)}$ : existe una ligera mayor especificidad y sensibilidad por parte de la EPDS con respecto a la BDI-II, en relación a la entrevista clínica.

El objetivo general de nuestro estudio fue determinar el comportamiento de ambos CA en una misma población gestante. Para esto, se evalúo la consistencia interna y el nivel de correlación existente entre ambos instrumentos.

\section{MÉTODOS}

Este estudio de tipo observacional transversal y analítico fue desarrollado en las instalaciones de la Unidad de Medicina
Fetal, sección monitoreo electrónico fetal del Instituto Nacional Materno Perinatal (INMP), en la ciudad de Lima, Perú. Se invitó a ingresar al estudio a todas las gestantes que acudieron a dicha sección durante los meses de mayo y junio de 2008, exceptuándose a las que presentaron un antecedente psiquiátrico diagnosticado. De un total de 810 gestantes invitadas a participar en el estudio y que fueron debidamente informadas sobre la investigación a realizar, 352 aceptaron, firmando libre y voluntariamente el consentimiento informado diseñado especialmente para este estudio, procediendo de inmediato al autollenado de los cuestionarios elaborados para este fin; se excluyó 45 cuestionarios debido a fallas en el llenado, quedando la muestra del estudio conformada por 307 gestantes. Se evalúo la presencia de síntomas depresivos mediante la escala de depresión posnatal de Edimburgo (EPDS), de 10 ítems ${ }^{(15)}$, y el inventario de la depresión de Beck (BDI-II), de 21 ítems ${ }^{(14)}$.

Para el registro de los datos, se construyó una base de datos en Microsoft Excel y, para el análisis de los mismos, se procedió

Tabla 1. Ítems utilizados en los dos únicos cuestionaros para la detección de síntomas depresivos en la población gestante.

\begin{tabular}{cl}
$\begin{array}{r}\text { Escala de depresión postnatal de } \\
\text { (EPDS, } 10 \text { ítems) }\end{array}$ \\
\hline 1. & Risa \\
2. & Disfrute \\
3. & Culpa \\
4. & Ansiedad \\
5. & Pánico \\
6. & Desesperanza \\
7. & Infelicidad \\
8. & Tristeza \\
9. & Llanto \\
10. & Ideación suicida
\end{tabular}

Inventario de la depresión de Beck (BDI-II, 21 ítems)

$\begin{array}{ll}\text { 1. } & \text { Tristeza } \\ \text { 2. } & \text { Pesimismo } \\ \text { 3. } & \text { Errores del pasado } \\ \text { 4. } & \text { Pérdida de placer } \\ \text { 5. } & \text { Sentimiento de culpa } \\ \text { 6. } & \text { Sentimiento de castigo } \\ \text { 7. } & \text { Disgusto por sí misma } \\ \text { 8. } & \text { Autocrítica } \\ \text { 9. } & \text { Deseos o pensamientos suicidas } \\ \text { 10. } & \text { Llanto } \\ \text { 11. } & \text { Inquietud } \\ \text { 12. Pérdida de interés } \\ \text { 13. Indecisión } \\ \text { 14. Inutilidad } \\ \text { 15. Pérdida de energía } \\ \text { 16. } \\ \text { 17. } & \text { Irritabilidad } \\ \text { 18. } & \text { Cambios en el apetito } \\ \text { 19. } & \text { Dificultad de concentración } \\ \text { 20. } & \text { Cansancio } \\ \text { 21. } & \text { Pérdida del interés sexual }\end{array}$

con el software estadístico SPSS-15. El análisis de las variables cuantitativas se realizó usando medidas de tendencia central y para las variables cualitativas se usó las distribuciones de frecuencias. Para determinar la consistencia interna de cada CA se utilizó el coeficiente alfa de Cronbach; se esperó que la consistencia interna de ambas escalas fuesen altas y que, por tanto, el valor del coeficiente alfa de Cronbach se encontrase entre 0,7 y $0,9^{(21,22)}$. La evaluación de la correlación entre la EPDS y la BDI-II se calculó mediante el coeficiente de correlación de Pearson; se consideró una correlación alta cuando el coeficiente se acercaba más a la unidad y, según el valor resultante, correlación alta $(0,8$ a 1$)$, moderada $(0,5$ a 0,79$)$ y baja (menor de 0,49 ).

\section{RESULTADOS}

El promedio de edad de las participantes del estudio fue 26 años, con un rango entre 14 y 46; todas las participantes cursaban el tercer trimestre de gestación y la edad gestacional promedio fue 37 semanas, con rango de 30 a 41 semanas.

La consistencia interna de la EPDS fue alta (coeficiente alfa de Cronbach 0,826), así como también la del BDI-II (coeficiente alfa de Cronbach 0,815).

El puntaje promedio del desarrollo del cuestionario EPDS fue 11,36; la distribución por ítems (Figura 1) permitió identificar como ítems con medias más altas a aquellos que evaluaron ansiedad, sentimiento de culpa, desesperanza, pánico, mientras que el ítem de menor valor fue el que hace referencia a ideación suicida.

El puntaje promedio del desarrollo del cuestionario BDI-II fue 15,96. La distribución por ítems (Figura 2) permitió identificar como ítems con medias más altas a: cambios del patrón de sueño, llanto, cansancio, inquietud; mientras que el ítem de menor valor, del mismo modo que con la EPDS, fue el que hace referencia a deseos o pensamientos suicidas.

Al evaluarse la correlación entre ambos CA, el coeficiente de correlación de Pearson entre la EPDS y la BDI-II fue 0,541 


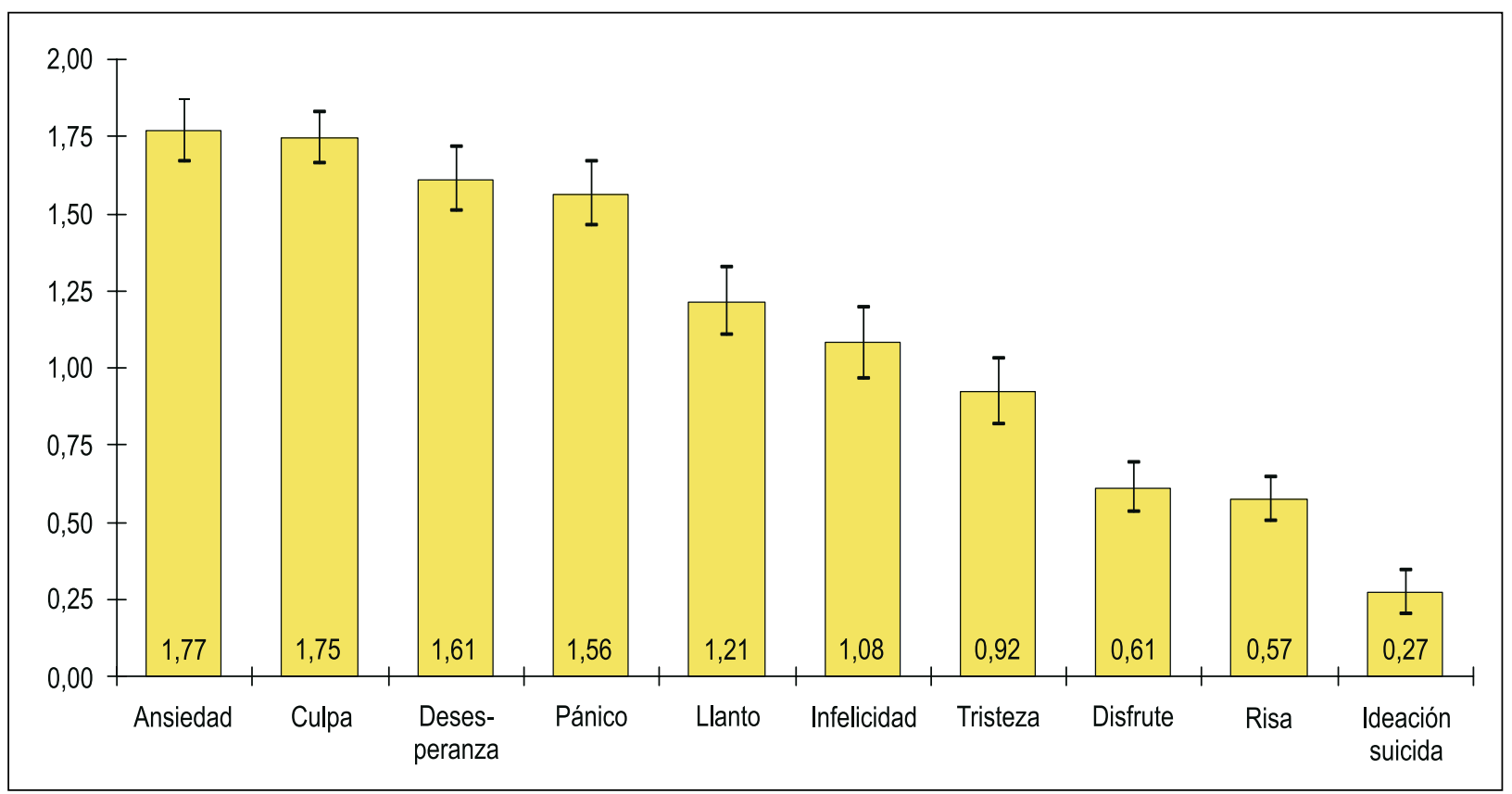

Figura 1. Distribución de medias para cada ítem de la escala de depresión posnatal de Edimburgo (EPDS).

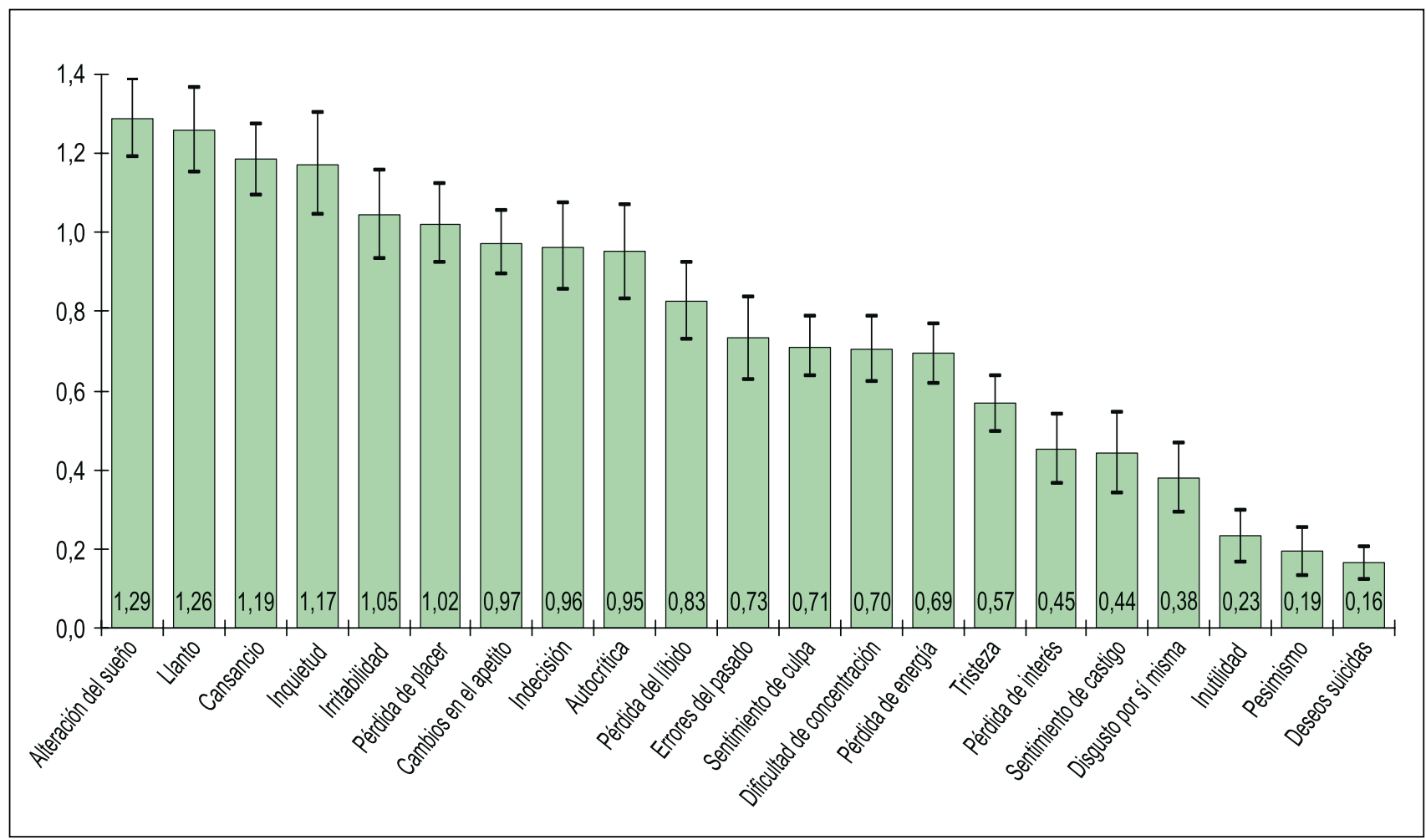

Figura 2. Distribución de medias para cada ítem de la depresión de Beck (BDI-II). 


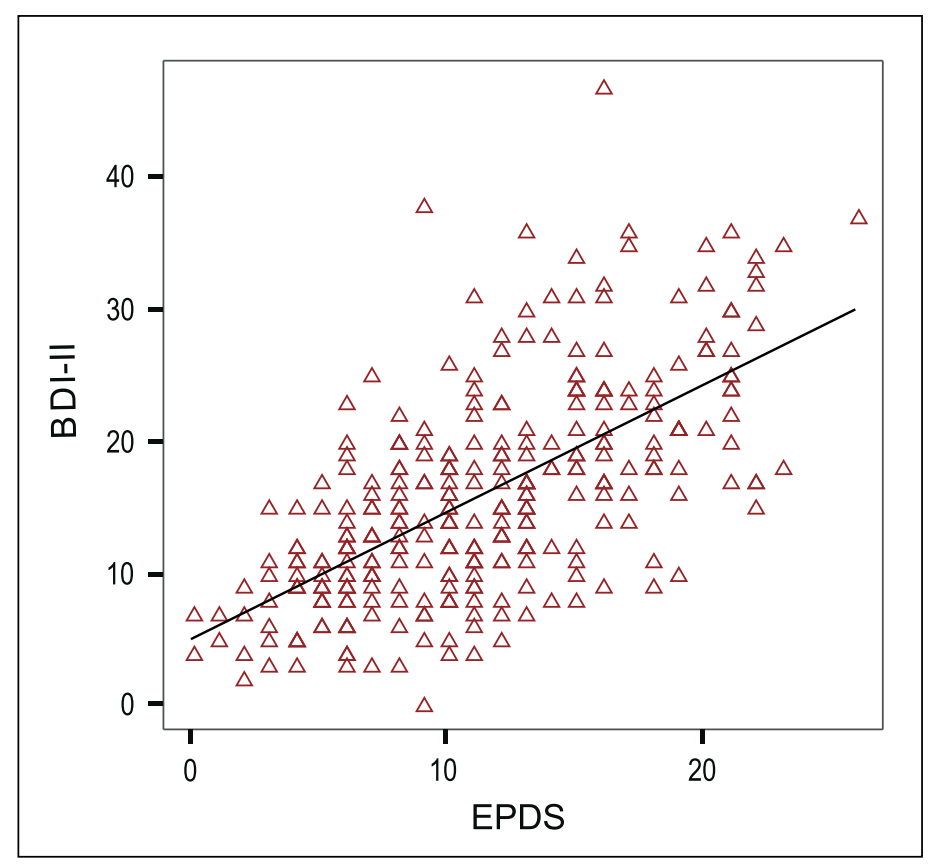

Figura 3. Gráfico de dispersión entre EPDS y BDI-II.

$(p<0,001)$, es decir que, entre ambos CA hubo una moderada correlación, aunque el grado de esta no fue alto (es decir: la unidad). Dicha correlación se evidencia en el gráfico de dispersión correspondiente (Figura 3).

\section{DISCUSIÓN}

Los resultados de la presente investigación muestran que tanto la EPDS como el BDI-II tienen una consistencia interna alta, lo que sugiere que ambos CA son confiables y posiblemente válidos para ser utilizados en la identificación de episodios depresivos en la población gestante de Lima, Perú.

En cuanto a la correlación entre ambos $\mathrm{CA}$, se puede ver que existe una moderada correlación (coeficiente de correlación de Pearson 0,541), lo cual permite afirmar que para efectos de tamizaje de posibles episodios depresivos se puede utilizar cualquiera de ellos. Consideramos que el grado de correlación no es mayor debido a que uno de los cuestionarios (BDI-II) toma en consideración ítems somáticofísicos, es decir, posibles falsos positivos, mientras que el otro cuestionario (EPDS) no los toma en cuenta; $y$, tal como se muestra en la Figura 2, de los 4 ítems con mayores medias en la BDI-II, el primero (cambios en el patrón de sueño) y el tercero (cansancio) son ítems de tipo somático-físicos. Se recomienda tener ello en consideración para futuros estudios a realizarse en este campo, con la finalidad de determinar una adecuada precisión o una alta probabilidad. Un cuestionario con síntomas potencialmente confusores podría tener mayor probabilidad de incluir todos los casos, lo cual podría ser beneficioso en estudios descriptivos, incluso exploratorios; mientras que un cuestionario que no considere dichos síntomas potencialmente confusores sea la mejor opción si lo que se pretende es un estudio analítico, por ejemplo donde se busquen factores causales, factores de riesgo y asociaciones mórbidas. Así, nuestros resultados refuerzan lo ya sugerido por otros investigadores ${ }^{(19,20)}$ : existiría mayor sensibilidad y especificidad con la EPDS que con la BDI-II.

En conclusión, los resultados del presente trabajo evidencian una consistencia interna alta de ambos cuestionarios así como una moderada correlación de estos tests evaluadores de síntomas depresivos en gestantes. Consideramos, como lo más resaltante, que sea con uno u otro cuestionario evaluado, los puntajes promedio son altos (11,36 para EPDS y 15,96 para BDI-II), lo que indicaría una alta probabilidad de trastornos depresivos en esta población. Las mujeres que participaron en este estudio se encontraban, como ya se refirió, recibiendo atención en la Unidad de Medicina Fetal del INMP, en donde, entre otras cosas, el único objetivo es determinar el estado de bienestar del feto. La evidencia existente ${ }^{(1-4)}$ permite sostener que no existirá jamás una verdadera determinación del estado de bienestar del feto si no se considera una evaluación del estado de la salud mental de la madre (en primera instancia el ecosistema más determinante para el nuevo ser). Por tanto, consideramos dicha evaluación como necesaria.

\section{REFERENCIAS BIBLIOGRÁFICAS}

1. Gold K, Dalton V, Schwenk T, Hayward R. What causes pregnancy loss? Preexisting mental illness as an independent risk factor. General Hospital Psychiatry. 2007;29:207-13.

2. Monk C. Stress and mood disorders during pregnancy: implications for child development. Psychiatric Quarterly. 2001;72(4):347-57.

3. Brouwers E, Van Baar A, Pop V. Maternal anxiety during pregnancy and subsequent infant development. Infant Behavior \& Development. 2001;24:95-106.

4. O'Connor T, Heron J, Golding J, Glover V, the ALSPAC Study Team. Maternal anxiety and behavioural/emotional problems in children: a test of a programming hypothesis. I Child Psychol Psychiatry. 2003;44:1025-36.

5. Faisal-Cury A, Menezes P. Prevalence of anxiety and depression during pregnancy in a private setting sample. Arch Womens Ment Health. 2007;10:25-32.

6. Vega J, Mazzotti G, Campos M. Validación de una versión en español de la Escala de Depresión Postnatal de Edimburgo. Actas Esp Psiquiatr. 2002;30(2):106-11.

7. Luna M, Otárola F, Almendras M, Jara L, Chuquizuta C. La depresión no respeta la gestación. Revista de Psiquiatría y Salud Mental Hermilio Valdizan. 2006;7(1):41-7.

8. Qiu Ch, Sanchez S, Lam N, Garcia P, Williams M. Associations of depression and depressive symptoms with preeclampsia: results from a Peruvian case-control study. BMC Women's Health. 2007;27:7-15

9. Sadek N, Nemeroff Ch. Actualización en neurobiología de la depresión. Revista de Psiquiatría del Uruguay. 2000;64(3):462-85.

10.Zandio M, Ferrín M, Cuesta M. Neurobiología de la depresión. Anales Sis San Navarra. 2002;25 (Supl 3):43-62. 
11. Kammerer A, Taylor A, Glover V. The HPA axis and perinatal depression: a hypothesis. Arch Womens Ment Health. 2006;9:187-96

12.0rtiz-Cabanillas P. Desarrollo formativo de la actividad psíquica personal. Paediatrica. 2004;6(1):29-41.

13. Holcomb W, Stone L, Lustmn P, Gavard J, Mostello D. Screening for depression in pregnancy: characteristics of the Beck Depression Inventory. Obstet Gynecol. 1996;8(6):1021-5.

14. Beck At, Steer Ra, Brown Gk. Manual for the Beck Depression Inventory-II. San Antonio, TX: Psychological Corporation, 1996.

15. Murray D, Cox Jl. Screening for depression during pregnancy with the Edinburgh depression scale (EPDS). J Reprod Infant Psychol. 1990;8:99107.

16. Adouard F, Glangeaud-Freudenthal N, Golse B. Validation of the Edinburgh postnatal depression scale (EPDS) in a sample of women with high-risk pregnancies in France. Arch Womens Ment Health. 2005;8:89-95.
17. Adewuya A, Ola B, Dada A, Fasoto 0. Validation of the Edinburgh Postnatal Depression Scale as a screening tool for depression in late pregnancy among Nigerian women. J Psychosom Obstet Gynaecol. 2006;27(4):267-72

18. Felice E, Saliba J, Grech V, Cox J. Validation of the Maltese version of the Edinburgh Postnatal Depression Scale. Arch Womens Ment Health. 2006;9:75-80.

19. Su KP, Chiu TH, Huang CL, Ho M, Lee CC, Wu PL, et al. Different cutoff points for different trimesters? The use of Edinburgh Postnatal Depression Scale and Beck Depression Inventory to screen for depression in pregnant Taiwanese women. General Hospital Psychiatry. 2007;29:436-41.

20. Bennett H, Einarson A, Taddio A, Koren G, Einarson T. Prevalence of depression during pregnancy: systematic review. Obstet Gynecol. 2004;103:698709.

21. Sánchez R, Echeverry J. Validación de escalas de medición en salud. Rev Salud Pública. 2004;6:302-18.
22. Campo-Arias A, Ayola-Castillo C, Peinado-Valencia H, Amor-Parra M, Cogollo Z. Escala de Edinburgh para depresión posparto: consistencia interna y estructura factorial en mujeres embarazadas de Cartagena, Colombia. Revista Colombiana de Obstetricia y Ginecología. 2007;58(4):277-83.

Manuscrito recibido el 15 de enero de 2009 y aceptado para publicación el 20 de febrero de 2009.

Correspondencia:

Mg. Nelly M. Lam Figueroa

Instituto de Investigaciones Clínicas

Hospital Nacional Dos de Mayo

Av. Grau cuadra 13. Parque Historia de la Medicina.

Lima 1, Perú.

Correo-e: nlamfigueroa@yahoo.es 